\title{
Compact Design of UHF RFID and NFC Antennas for Mobile Phones
}

\author{
F.Paredes ${ }^{1 *}$, I. Cairó ${ }^{1}$, S. Zuffanelli ${ }^{1}$, G. Zamora ${ }^{1}$, J. Bonache ${ }^{1}$ and F. Martin ${ }^{1}$ \\ ${ }^{1}$ Department of Electronic Engineering, Universitat Autònoma de Barcelona, Bellaterra, \\ Spain \\ *ferran.paredes@uab.es
}

\begin{abstract}
A small size and low profile antenna has been developed in order to provide any mobile phone with UHF RFID reader functionality. For that purpose, a patch antenna topology has been chosen on account of the mobile phone battery, which exhibits an electromagnetic behavior similar to a metal plane. The low profile patch antenna has been designed to operate at the European UHF RFID band. In order to communicate the mobile phone with the RFID reader module, NFC technology has been considered. Thus, an NFC antenna, based on square coils, operating at $13.56 \mathrm{MHz}$ has been also designed. Such antenna has been etched on the opposite side of the patch antenna. Overall dimensions of the prototype are $60 \mathrm{~mm} \times 100 \mathrm{~mm}$, i.e., small enough to fit the dimensions of a mobile phone. As proof of concepts to evaluate the performance of the designed antennas, an UHF RFID reader module and a NFC reader module are tested. The measured read range reaches up to $1 \mathrm{~m}$ for some commercial tags.
\end{abstract}

\section{Introduction}

The applications of radiofrequency identification (RFID) [1] have increased in recent years, but not as fast as expected. The forecasts anticipated that RFID would be largely employed in the ultra-high frequencies (UHF RFID) due to the reduction of tag's price [2]. The efforts improving the ASIC (or chip) performance $[3,4]$, i.e. sensitivity and input impedance (allowing for increased read ranges and operation bandwidth), as well as the antenna performance [5], have been significant. Indeed, most current research articles are focused on antenna performance, and such papers present different configurations of dipole and monopoles, which usually present some degree of meandering in order to reduce dimensions $[6,7]$ yet keeping suitable read ranges.

Nowadays, the price of tags is already affordable and the next challenge is to reduce the price of readers. The concept proposed in this work consists of taking advantage of mobile phones to spread the RFID technology, by developing a low profile patch antenna. The integration of an UHF RFID reader with the mobile phone was already investigated [8,9], reaching distances up to 40 and $60 \mathrm{~cm}$, respectively, with $100 \mathrm{~mW}$ of transmitted power. In [10], the UHF RFID functionality was achieved by modifying the mobile phone operating system. However, the read range was limited to $5 \mathrm{~cm}$, even by using the maximum output power supplied by the mobile phone $(0.25 \mathrm{~W})$. Other proposed non-embedded solution consisted of 


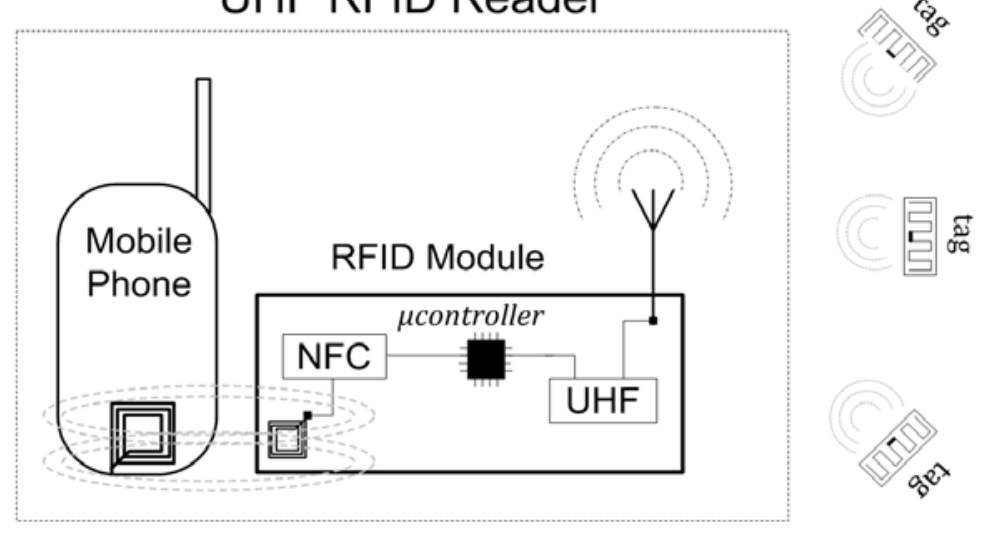

Fig. 1. Diagram of UHF RFID reader, which consists of a mobile phone communicated with the RFID module by means of NFC technology. This configuration takes advantage of mobile phones to detect UHF RFID tags.

a functional cover [11] which only worked for a specific mobile phone (Nokia E61i), and the read range was limited to $50 \mathrm{~cm}$. Another related work is found in [12] where an UHF RFID reader was developed and connected by USB to a mobile phone, although the read range was not mentioned.

This work explores the possibility of extending the UHF RFID functionality to any mobile phone or device equipped with NFC technology, with special focus on increasing the read range in comparison to the state of the art. The goal of this paper is to design a low profile patch antenna for an UHF RFID module able to operate in contact with a mobile phone. The communication between the mobile phone and such a module is achieved by means of near field communication (NFC) technology [13], since most smart phones, tablets, etc. are equipped with NFC capability (see Fig. 1). For this purpose, a design of an NFC antenna is also carried out, and the whole system is tested separately with NFC and UHF RFID communication readers.

\section{UHF RFID and NFC Antenna Design}

\subsection{UHF RFID Antenna Design}

The back side of mobile phones is characterized by the presence of a battery, which essentially behaves as a metal plane [14], and consequently, the use of dipoles is not convenient [15]. Thus, a microstrip patch is one of the most suitable configuration to be used as UHF RFID antenna. Moreover, a common characteristic of such antennas is that they are low profile [16], allowing for a narrow design of the UHF RFID module. The size of the antenna, namely $60 \mathrm{~mm} \times 100 \mathrm{~mm}$, was chosen to fit to typical 
commercial smart phones. Since the abovementioned size is smaller than half-wavelength at the UHF RFID band, a quarter wavelength patch antenna was considered. In order to reduce the antenna length even more, the rectangular patch was bent to get a right-angle (see Fig. 2). The feeding line was inserted inside the horizontal section of the patch antenna to properly excite the fundamental mode along the $y$ direction, thus forcing linear polarization. The antenna was designed on a Rogers RO4003 substrate with thickness $h=0.8 \mathrm{~mm}$, dielectric constant $\varepsilon_{r}=3.55$, and loss tangent $\tan \delta=0.0022$. The short circuits to ground were implemented by using vias. The dimensions of the antenna layout, shown in Fig. 2, are $w_{1}=0.4 \mathrm{~mm}, l_{1}=12.6 \mathrm{~mm}, w_{2}=1.5 \mathrm{~mm}, l_{2}=9.5 \mathrm{~mm}, w_{3}=1.5 \mathrm{~mm}, l_{3}=10.2 \mathrm{~mm}, w_{4}=5 \mathrm{~mm}, l_{4}=8.9 \mathrm{~mm}$, $w_{5}=3.8 \mathrm{~mm}, W=23.8 \mathrm{~mm}, L=45 \mathrm{~mm}$. It can be observed that a significant size reduction $\left(\lambda_{0} / 7.5\right)$ is achieved as compared to typical half-wavelength patch antennas. The radiation efficiency was found to be $20 \%$ and the input impedance $Z_{i n_{-} A N T}=1.45-\mathrm{j} 22 \Omega$ at $867 \mathrm{MHz}$. Therefore, an impedance matching network is required to match the UHF RFID antenna to the $50 \Omega$ impedance of the UHF RFID reader. The proposed microstrip matching network consists of a shunt short-circuited stub connected in cascade to a quarter-wavelength transmission line. The short-circuited stub has a characteristic impedance of $Z_{\text {stub }}=57.5 \Omega$ and electrical length $\beta l_{\text {stub }}=15^{\circ}$, which corresponds to a width of $w_{2}=1.5 \mathrm{~mm}$ and length $l_{2}=9.5 \mathrm{~mm}$, and provides an impedance of $Z_{\text {in_Stub }}=\mathrm{j} 15.5 \Omega$. After setting the shunt impedance, the required impedance of the quarter wavelength transmission line was found to be $Z_{\lambda / 4}=102 \Omega$. The length and width dimensions of the line are $l_{l}{ }^{\prime}=55 \mathrm{~mm}$ and $w_{l}=0.4 \mathrm{~mm}$, respectively. In order to reduce dimensions, the impedance matching network was meandered $\left(l_{1}=12.6 \mathrm{~mm}\right)$, as shown in Fig. 2 . The electromagnetic simulation of the power reflection coefficient is plotted in Fig. 3, showing good impedance matching $(-23 \mathrm{~dB}$ at $867 \mathrm{MHz})$. The simulated radiation pattern of the patch antenna, taking into account the impedance matching network, is shown in Fig. 4. Both the E-plane (yz-plane) and the Hplane ( $x z$-plane) were normalized to the maximum antenna gain $(G=-3.1 \mathrm{~dB})$ exhibited at broadside direction, which corresponds to a radiation efficiency of $\eta_{\mathrm{rad}}=18 \%$. 


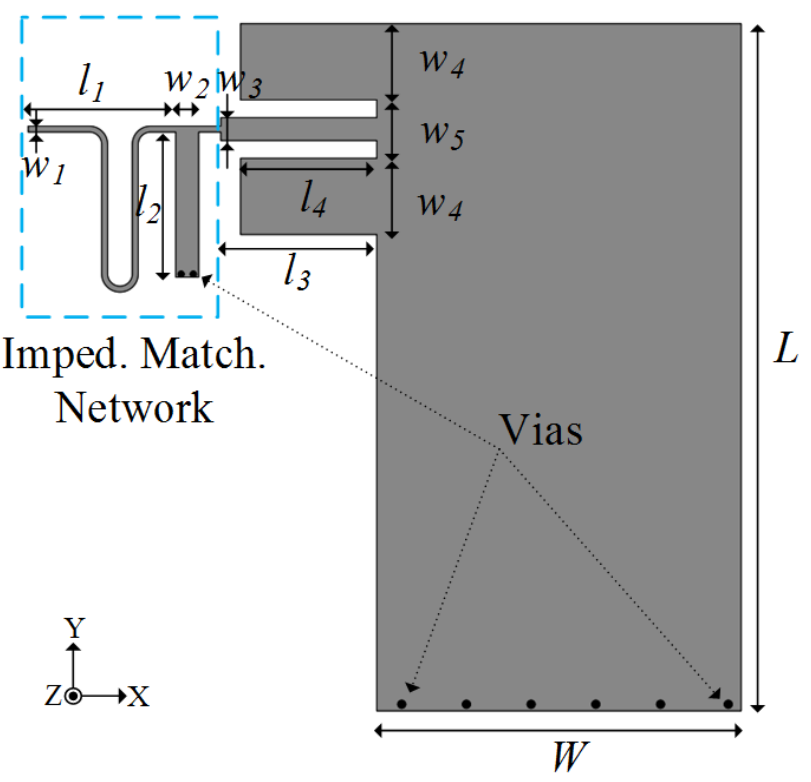

Fig. 2. Layout of the UHF RFID reader antenna, including the meandered impedance matching network.

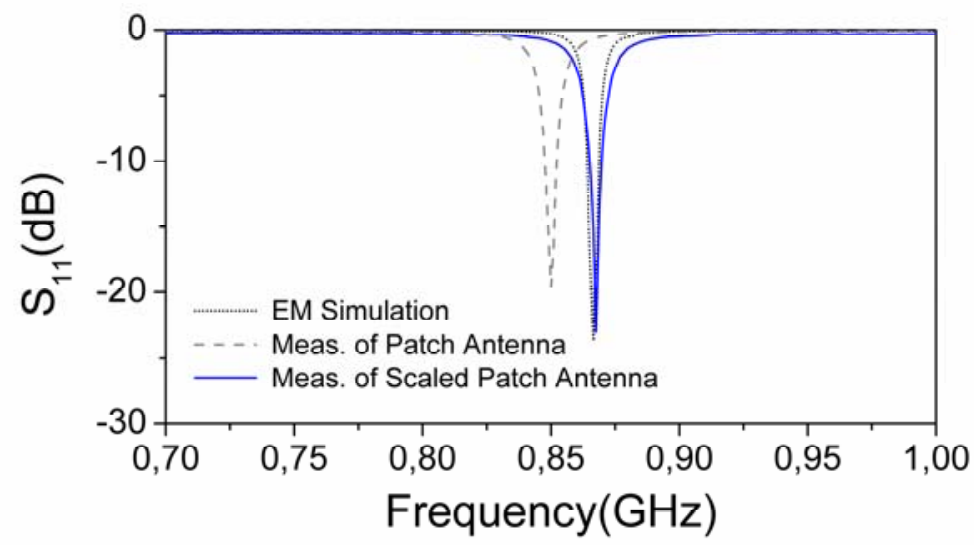

Fig. 3. Simulated and measured frequency responses of the UHF RFID reader antenna

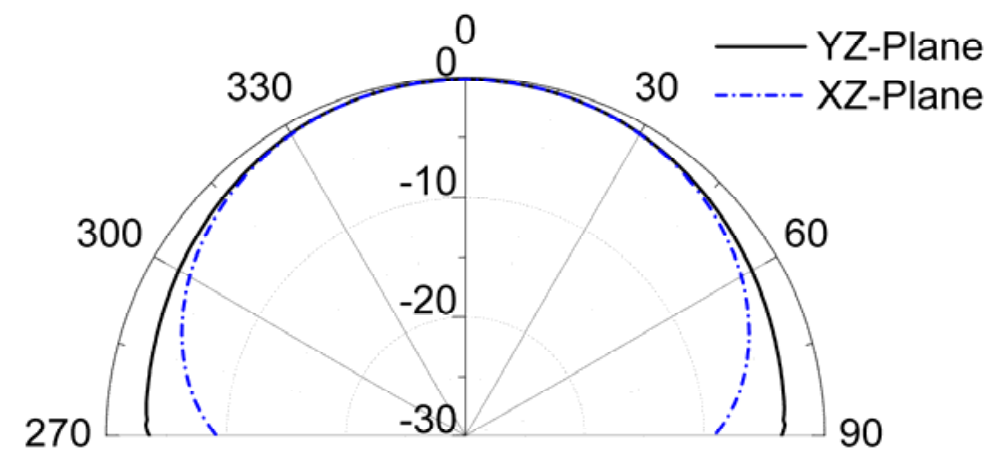

Fig. 4. Normalized radiation diagram for the UHF RFID reader antenna 


\subsection{NFC Antenna Design}

Loop antennas in NFC systems are intended to resonate at the operation frequency $(13.56 \mathrm{MHz})$ with the output stage of the reader, so that the antenna input inductance is the most important parameter. In our case, we used the $A S 3911$ NFC reader [17] mounted on a demonstration board provided by the manufacturer, where the required antenna reactance is $X_{\mathrm{A}}=\mathrm{j} 40 \Omega$. The proposed antenna is based on planar square coils, following the designs of $[18,19]$ and consists of three turns of $30 \mathrm{~mm} \times 30 \mathrm{~mm}$ with an access line of $5 \mathrm{~mm}$ to connect the inner and the outer paths of the square coil (see bottom face of Fig. 6b). The strip width is $0.5 \mathrm{~mm}$ and the gaps between turns are $0.5 \mathrm{~mm}$. The antenna was embedded in the bottom layer of the substrate containing the UHF RFID antenna, in a dedicated area obtained by removing the patch ground plane. The simulated antenna input impedance is shown in Fig. 5, which suggests a reactance of j40 $\Omega$ and a very low input resistance at the operation frequency. A wider frequency range is also shown in Fig. 5 (in the inset), where the coil self-resonance can be observed.

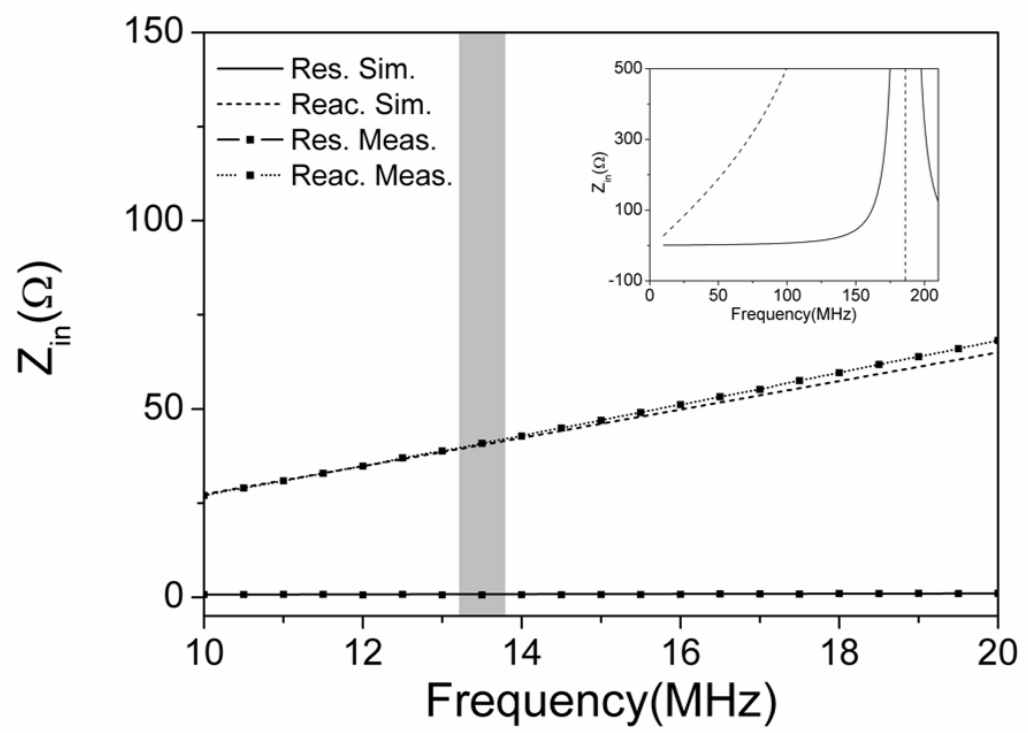

Fig. 5. Simulated and measured input impedance of NFC reader antenna.

\section{Fabrication and Measurements}

The prototype, which includes the UHF RFID antenna on the upper side of the substrate (Fig. 6a), and the NFC antenna on the lower side of the substrate (Fig. 6b), was fabricated with a milling machine. The return loss $S_{11}$ of the UHF RFID antenna was measured by means of the $E 8364 B$ vector network analyzer. As it can be seen in Figure 3, a frequency shift of $20 \mathrm{MHz}$ occurred, with a non-significant 
degradation of the impedance matching. The UHF RFID antenna was scaled a $2 \%$ down, which according to electromagnetic simulation is expected to keep the performance, in order to compensate for the frequency shift. Thus, the full prototype, including the NFC antenna, was fabricated again and measured (Fig. 3 and Fig. 5 for the UHF RFID and the NFC antenna, respectively). The overall prototype dimensions are $60 \mathrm{~mm}$ and $100 \mathrm{~mm}$ for the width and length, respectively.

A link budget was used to measure the RFID antenna radiation patterns of the E-plane (yz-plane) and H-plane ( $x z$-plane), including co-polar and cross-polar components. The link budget consisted of the abovementioned vector network analyzer, where the first port was connected to an $83006 \mathrm{~A}$ amplifier. The output of the amplifier was connected to an ETS-Lindgren 3164-07 horn antenna, which was located inside an anechoic chamber. The antenna prototype under test was also located in the anechoic camber and connected to the second vector network analyzer port. In order to measure different angles, an angular sweep was carried out by means of a stepper motor and a controller, and it allowed obtaining the radiation diagram depicted in Fig. 7. The measured maximum realized gain $G_{t}=-3.7 \mathrm{~dB}$ is reached at $-14^{\circ}$ from the broadside direction in the $x z$-plane. From the measured cross-polar pattern, it can be observed that linear polarization is achieved around the broadside direction.
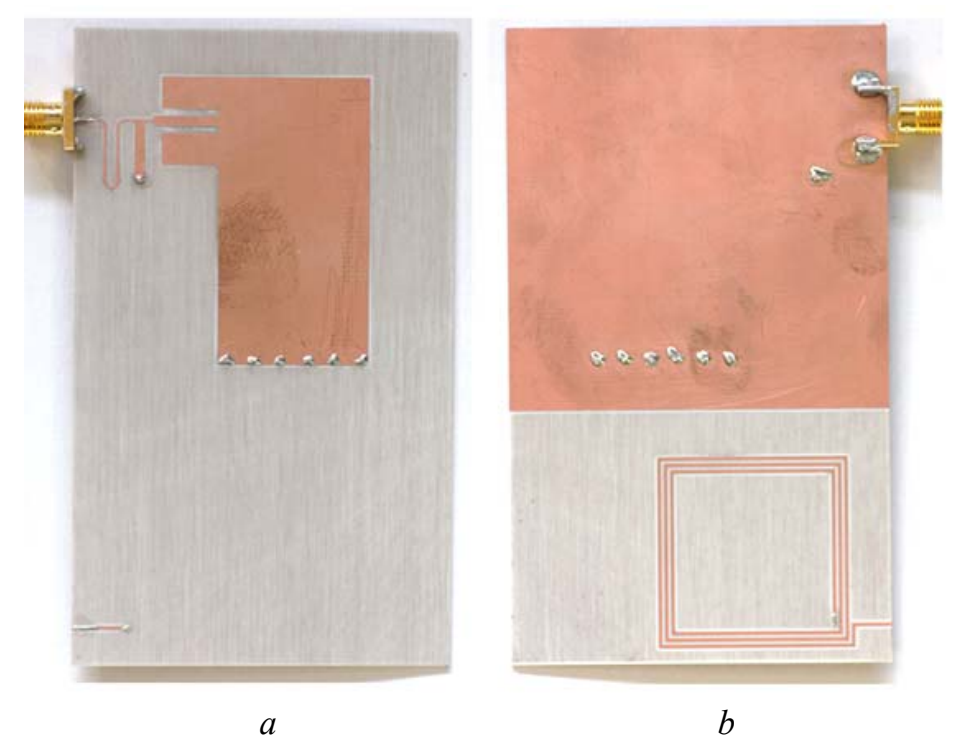

Fig. 6. Final prototype considering the UHF RFID reader patch antenna and the NFC square coil antenna. a Top view

b Bottom view 


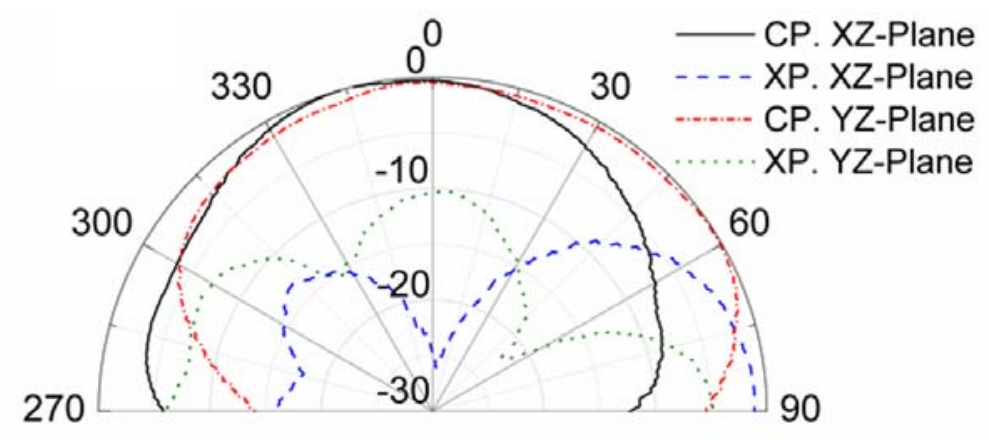

Fig. 7. Measured normalized radiation diagram for the UHF RFID antenna shown in Fig. 6. The CP and XP stand for copolar and cross-polar components, respectively.

In order to test both antennas, the NFC reader (AS3911 [17]) and the UHF RFID reader (AS3993 [20]) were connected to the prototype. Both readers were powered via USB with a laptop, where specific test software was run. Contact communication between the NFC reader and the mobile phone was successfully established. In order to test the NFC communication performance, the prototype was separated from the mobile phone until the NFC communication was lost (roughly $2 \mathrm{~cm}$ ). However, for the current application, it is preferred to locate the prototype in contact to the back side of the mobile phone to ensure permanent communication. After the NFC communication was validated, the UHF RFID functionality was also tested. According to the Friis free space formula [5], the read range can be calculated as

$$
R R=\frac{\lambda}{4 \pi} \sqrt{\frac{\tau G_{r} P_{t} G_{t}}{P_{t h}}}
$$

where $\lambda$ is the wavelength, $\tau$ is the power transmission coefficient, $P_{t h}$ is the minimum threshold power to activate the tag ASIC, $G_{r}$ is the receiving tag antenna gain, $P_{t}$ is the power transmitted by the reader and $G_{t}$ is the realized gain of the transmitting antenna. Thus, the link budget calculation was performed as follows. The output power from the reader is roughly $16 \mathrm{dBm}$, taking into account the abovementioned UHF RFID module $\left(P_{t}=20 \mathrm{dBm}\right)$ and the designed antenna realized gain $\left(G_{t}=-3.7 \mathrm{~dB}\right)$. On the other hand, the input power for typical commercial tags is expected to be nearly $-18 \mathrm{dBm}$, considering a chip sensitivity of $-17 \mathrm{dBm}$ and a receiving tag antenna realized gain of $1 \mathrm{~dB}$ ). The difference between the reader output power and the tag input power, which accounts for the path loss, results in $-34 \mathrm{~dB}$. According to (1), this value corresponds to a read range of $1.4 \mathrm{~m}$. Experimental validation was carried out in a real scenario by means of the ALN-9640 Squiggle tag inlay [21], and $1 \mathrm{~m}$ of read range was reached 
at broadside direction. Such distance is high enough to validate the presented solution, as well as to improve the state of the art. Furthermore, it was checked that the NFC communication keeps working while the UHF RFID module is connected to the patch antenna, as well as the UHF RFID module still works while the mobile phone is operating, verifying that there are non-significant electromagnetic interferences between devices $[22,23]$.

\section{Conclusion}

A novel strategy to provide UHF RFID reader capabilities to a mobile phone has been proposed in this work. A compact low profile UHF RFID patch antenna has been designed to be fitted within the size of typical commercial mobile phones. An NFC antenna has also been designed to allow for a wireless communication between the mobile phone and the RFID module. Both antennas have been manufactured in a prototype of reduced dimensions $(60 \mathrm{~mm} \times 100 \mathrm{~mm})$ and have been tested by a respective NFC and UHF RFID communication reader modules. The results have shown a successful communication between the NFC antenna and the mobile phone. Moreover, an experimental read range of $1 \mathrm{~m}$ was obtained when the UHF RFID antenna has been employed with commercial tags. This read range represents a significant improvement over the state of the art.

Future work comprises the integration of the NFC and RFID communication reader modules, which will be managed by a microcontroller, into the same prototype along with antennas and a slim battery required to power the whole system.

\section{Acknowledgments}

This work has been supported by MINECO-Spain (project TEC2013-40600-R), by Generalitat de Catalunya (project 2014SGR-157) and by FEDER funds. Ferran Martín has been awarded with an ICREA Academia distinction.

The authors are with CIMITEC, Department of Electronic Engineering, Universitat Autònoma de Barcelona, 08193 Bellaterra, Spain. 


\section{References}

[1] Finkenzeller, K.: 'RFID Handbook: Radio-Frequency Identification Fundamentals and Applications', (Wiley, 2004, 2nd edn)

[2] Glidden, R., Bockorick, C., Cooper, S., et al.: 'Design of ultra-low cost UHF RFID tags for supply chain applications', IEEE Com. Mag., 2004, 42, pp. 140-151

[3] Bergeret, E., Gaubert, J., Pannier P., et al.: 'Modeling and design of CMOS UHF voltage multiplier for RFID in a EEPROM compatible process', IEEE Trans. Circuits Syst., 2007, 54, pp. 833-837

[4] Tran, N., and Lee, J.W.: 'Simple high-sensitivity voltage multiplier for UHF-band semi-passive radiofrequency identification tags using a standard CMOS process', IET Microwave, Antennas \& Propagation, 2010, 4, pp. 19741979

[5] Rao, K.V.S., Nikitin, P.V., and Lam, S.F.: 'Antenna design for UHF RFID tags: a review and a practical application', IEEE Trans. Ant. Prop., 2005, 53, pp. 3870-3876

[6] Marrocco, G.: 'The art of UHF RFID antenna design: impedance-matching and size-reduction techniques', IEEE Ant. Propag. Mag., 2008, 50, pp. 66-79

[7] Abdulhadi, A.E., Abdhari, R.: 'Design and experimental evaluation of miniaturized monopole UHF RFID tag antennas', IEEE Ant. Wirel. Prop. Let, 2012, 11, pp. 248-251

[8] Pursula, P., Seppa, H.: 'Hybrid transformer-based adaptive RF front end for UHF RFID mobile phone readers'. Proc. IEEE Int. Conf. on RFID, Las Vegas, USA, April 2008, pp. 150-155

[9] Kwon, I., Eo, Y., Bang, H., et al: 'A single-chip CMOS Transceiver for UHF mobile RFID reader', IEEE Journ. Solid-State Circ., 2008, 43, pp.729-738

[10] Zhang, P., and Ma, G.F.: 'Design of passive RFID reader based on mobile phone'. Proc. 2nd Int. Conf. Networks Security Wireless Communications and Trusted Computing (NSWCTC), Hubei, China, April 2010, pp. 446-449

[11] Savolainen, J.T., Hirvola, H., and Iraji, S.: 'EPC UHF RFID reader: mobile phone integration and services'. Proc. 6th IEEE Consumer Communications and Networking Conf. (CCNC), Nevada, USA, Jan. 2009, pp. 1-5 
[12] Loeffler, A., and Heisler, S.: 'An UHF RFID reader for cell phones'. Proc. IEEE Int. Conf. Wireless Information Technology and Systems (ICWITS), Honolulu, USA, August 2010, pp. 1-4

[13] Coskun, V., Ok, K., and Ozdenizci, B.: 'Near Field Communication (NFC): From theory to Practice', (Wiley, 2011, 1st edn)

[14] Linden, D., and Reddy, T.B.: 'Handbook of Batteries', (McGraw-Hill, 2001, 3rd edn.)

[15] Xie, Z.M., Lai, X.Z., and Hu, R.J.: 'Compact UHF RFID reader antenna using bended fold dipole structure for mobile RFID system'. Proc. Int. Conf. Mach. Lear. Cybernetics, Guilin, China, July 2011, pp. 414-417

[16] James, J.R.: 'Handbook of Microstrip Antennas', (IEE Electromagnetic Wave Series 28, 1989)

[17] 'AMS AS3911 NFC/HF Reader datasheet', http://ams.com/eng/Products/WirelessConnectivity/Readers/AS3911B, accessed June 2016

[18] Basat, S.S., Lim, K., Laskar, J., et al.: 'Design and modeling of embedded 13.56 MHz RFID antennas'. Proc. IEEE Int. Symp. Ant. and Prop. Society, Washington, USA, July 2005, pp. 64-67

[19] Nummela, J., Ukkonen, L., Sydanheimo, L., et al.: '13.56 MHz RFID antenna for cell phone integrated reader'. Proc. IEEE Int. Symp. Ant. and Prop. Society, Honolulu, USA, June2007, pp. 1088-1091

[20] 'AMS AS3993 UHF RFID Reader datasheet', $\underline{\text { http://ams.com/eng/Products/Wireless- }}$ Connectivity/Readers/AS3993, accessed Nov. 2015

[21] 'Alien squiggle inlay ALN-940 tag datasheet', http://www.alientechnology.com/wp-content/uploads/AlienTechnology-Higgs-3-ALN-9640-Squiggle.pdf, accessed Feb. 2014

[22] Arnaud-Cormos, D., Letertre, T., Diet, A., et al.: 'Electromagnetic environment of RFID systems'. Proc European Microwave Conf., Munich, Germany, Oct. 2007, pp. 1652-1655

[23] Hong, W., Kan, R., and Li, S.: 'Electromagnetic Compatibility of UHF RFID to GSM'. Proc. Int. Symp. Electromagnetic Compatibility (EMC), Qingdao, China, Oct. 2007, pp. 63-66 